\title{
ORIGINAL ARTICLE Is floral divergence sufficient to maintain species boundaries upon secondary contact in Mediterranean food-deceptive orchids?
}

\begin{abstract}
A Zitari ${ }^{1,2}$, G Scopece $^{1}$, AN Helal ${ }^{2}$, A Widmer $^{3}$ and S Cozzolino ${ }^{1}$
Analyzing the processes that determine whether species boundaries are maintained on secondary contact may shed light on the early phase of speciation. In Anacamptis morio and Anacamptis longicornu, two Mediterranean orchid sister-species, we used molecular and morphological analyses, together with estimates of pollination success and experimental crosses, to assess whether floral isolation can shelter the species' genomes from genetic admixture on secondary contact. We found substantial genetic and morphological homogenization in sympatric populations in combination with an apparent lack of postmating isolation. We further detected asymmetric introgression in the sympatric populations and an imbalance in cytotype representation, which may be due either to a difference in flowering phenology or else be a consequence of cytonuclear incompatibilities. Estimates of genetic clines for markers across sympatric zones revealed markers that significantly deviated from neutral expectations. We observed a significant correlation between spur length and reproductive success in sympatric populations, which may suggest that directional selection is the main cause of morphological differentiation in this species pair. Our results suggest that allopatric divergence has not led to the evolution of sufficient reproductive isolation to prevent genomic admixture on secondary contact in this orchid species pair.
\end{abstract}

Heredity (2012) 108, 219-228; doi:10.1038/hdy.2011.61; published online 27 July 2011

Keywords: allopatric speciation; divergent selection; genetic admixture; genetic clines; Orchidaceae; reproductive isolation

\section{INTRODUCTION}

Geographic separation and repeated range contractions and expansions as consequences of paleo-climatic events have had a strong influence on current plant species diversity and distribution (Hewitt, 2004), because the recurring formation of geographically isolated populations may drive allopatric speciation (Coyne and Orr, 2004). In the absence of gene flow, allopatric populations may diverge by fixation of mutations as a consequence of random genetic drift, which may be enhanced by repeated bottlenecks associated with range contractions. This may lead to the fixation of chromosomal rearrangements, a biological process that is generally considered of primary importance for plant speciation (Rieseberg and Willis, 2007). Alternatively, allopatric populations may diverge as a consequence of selection (Rundle and Nosil, 2005). Range expansions of formerly isolated lineages during warmer periods often lead to the formation of secondary contact zones in which previously allopatric populations are exposed to interspecific mating (Hewitt, 2001) and, when they are at least partially interfertile, to hybridization. In this case, the maintenance of species integrity in sympatry implies the existence of a dynamic balance between the production of hybrids and their elimination by natural selection (Harrison, 1993). This balance depends on the types and strengths of reproductive barriers that have arisen during geographical isolation (Barton and Hewitt, 1985). In those cases in which isolating mechanisms did not evolve in allopatry or were not sufficiently strong to maintain species integrity on secondary contact, gene flow can lead to the homogenization of differences between formerly allopatric populations (Taylor et al., 2006).

Secondary contact zones between recently diverged lineages are thus of primary interest in the study of the speciation process because an analysis of the processes that determine whether the delicate integrity of incipient species that lack complete reproductive isolation can be maintained or not, may shed light on the factors and processes that initiate speciation (Scopece et al., 2010). In allopatric plant populations, speciation can occur after fixation of chromosomal changes or can be directly or indirectly caused by divergent selection that can drive morphological divergence of flowers through adaptation to different pollinators or to local ecological conditions (Grant, 1981). In the latter case, a shift to different pollinators directly causes a form of premating isolation (Armbruster and Muchhala, 2009) while, in the former case, the evolution of local adaptation can accelerate the accumulation of epistatic interactions among locally adapted gene complexes (Schluter, 2000; Gavrilets, 2003) and can produce, as by-product, the evolution of postzygotic isolation mechanisms (Bateson-Dobzhansky-Muller model: Coyne and Orr, 2004). There are good examples of reproductive isolation based on BatesonDobzhansky-Muller incompatibilities (Bomblies and Weigel, 2007 and references therein) but the importance of these genetic mechanisms in plant speciation has been challenged because of the observation that, contrary to chromosomal changes, epistatic interactions are often polymorphic within species (reviewed in Scopece et al., 2010)

${ }^{1}$ Department of Structural and Functional Biology, University Federico II of Naples, Naples, Italy; ${ }^{2}$ Genome, Diagnostic and Valorisation, Institute of Biotechnology, Monastir, Tunisia and ${ }^{3}$ ETH Zurich, Plant Ecological Genetics, Institute of Integrative Biology, Zurich, Switzerland

Correspondence: Dr G Scopece, Department of Structural and Functional Biology, University Federico II of Naples, via Cinthia, Naples I-80126, Italy. E-mail: giosco11@fastwebnet.it

Received 19 January 2011; revised 17 May 2011; accepted 14 June 2011; published online 27 July 2011 
and often make a small contribution to total isolation (Rieseberg and Willis, 2007).

In this study, we focus on two closely related species of fooddeceptive Mediterranean orchids, Anacamptis morio and Anacamptis longicornu. Reproductive barriers in this orchid group have been investigated in much detail in recent years (reviewed in Cozzolino and Widmer, 2005a; Cozzolino and Scopece, 2008). These studies revealed that hybrid zones among Mediterranean food-deceptive orchids are typically dominated by $\mathrm{F} 1$ hybrids and often contain only few backcross individuals (reviewed in Cozzolino and Widmer, 2005b). This hybrid zone structure suggests that gene flow is limited between species pairs. The two related species A. morio and Anacamptis papilionacea, for example, were found to be completely reproductively isolated as a consequence of a strong hybrid sterility barrier (Moccia et al., 2007) but only by a weak premating barrier (Scopece et al., 2009). Similar findings of very strong (or complete) postzygotic isolation were also reported among other related species pairs, despite weak prezygotic barriers (reviewed in Cozzolino and Widmer, 2005b).

A comparative study has further shown that, contrary to general expectations in orchids, species boundaries in food-deceptive orchids are maintained primarily by postmating isolation, which includes both pre- and postzygotic barriers (Scopece et al., 2007). In contrast, premating barriers are incomplete because of weak pollinator specialization. Consequently, allopatric speciation has been suggested to be the most important speciation mechanism in these Mediterranean orchids (Cozzolino et al., 2004; Cozzolino and Scopece, 2008). This hypothesis was further supported by the observation that closely related food-deceptive orchid species often have asymmetric karyotypes (Cozzolino et al., 2004) or even different chromosome numbers (D’Emerico et al., 1996; Moccia et al., 2007), which may indicate that reproductive isolation evolves most likely as a consequence of the fixation of chromosomal changes in allopatric populations (Cozzolino et al., 2004), rather than by the gradual accumulation of negative epistatic interactions. However, identifying the mechanisms that cause speciation can best be done at an early stage of divergence, before speciation has gone to completion.

The two investigated taxa, A. morio and A. longicornu differ in floral traits related to pollination and have a typical Mediterranean vicariant distribution (Delforge, 2005), suggesting a phase of allopatric isolation long enough to allow for the establishment of the observed morphological differences. The distribution ranges overlap only in Sicily and Corsica where the two species form hybrid zones in which the assignment of individuals to parental species can be difficult. Despite their clear divergence in morphology (in particular spur length) and ribosomal DNA sequence (Cafasso et al., 2001), these two taxa show very similar karyotypes (D'Emerico et al., 1996). The majority of Mediterranean food-deceptive orchids that occur in sympatry have divergent karyotypes that cause strong postzygotic isolation and prevent extensive hybridization and introgression (Cozzolino et al., 2004). In contrast, these two species allow us to assess if, in absence of major chromosomal differences, other forms of reproductive isolation (both pre- and postmating) shelter their genomes from merging on secondary contact.

In this study, we specifically addressed the following questions:

(i) What are the genetic and phenotypic patterns of hybrid zones between A. morio and A. longicornu?

(ii) What form of pre- or postmating (both pre- or postzygotic) reproductive isolation has evolved in allopatry between these taxa? (iii) Are the two taxa sufficiently reproductively isolated to maintain their integrity on secondary contact?

The finding of extensive gene flow and of morphological and genetic homogenization would indicate that floral isolation alone is not sufficient to maintain species boundaries among Mediterranean food-deceptive orchids on secondary contact.

\section{MATERIALS AND METHODS}

\section{Study system}

A. morio and A. longicornu are two closely related taxa of subtribe Orchidinae. A. morio is widespread and relatively common from Northern Europe (Southern Norway) to the northern borders of the Mediterranean basin, while A. longicornu has a restricted distribution and occurs in parts of the southwestern border of the Mediterranean basin (Tunisia and Algeria) and on Sicily, Sardinia, Corsica and Balearic islands. The only areas where the two taxa are known to coexist are eastern Sicily and southern Corsica where they form hybrid zones (Delforge, 2005). Despite the known formation of hybrids, morphologically clearly differentiated populations of the two taxa that grow in geographic isolation (allopatry) are also present on Sicily. In this study, we analyzed both sympatric populations that are involved in hybrid formation, and allopatric populations of parental species. In detail, for A. longicornu we distinguished allopatric populations from eastern Sicily (hereafter referred to as close allopatric A. longicornu), as well as geographically distant populations from western Sicily and from Tunisia (hereafter referred to as distant allopatric A. longicornu). Similarly, for A. morio we distinguished allopatric populations from eastern Sicily (hereafter referred to as close allopatric A. morio), as well as geographically distant populations from Southern Italy (hereafter referred to as distant allopatric A. morio).

\section{Plant material}

During the flowering seasons of 2005, 2006 and 2008, we surveyed 12 natural orchid populations ( 2 sympatric populations, 2 distant allopatric and 2 close allopatric populations of A. morio, 4 distant allopatric and 2 close allopatric populations of A. longicornu; Table 1 for details).

For morphological analyses, we measured floral traits from 277 individuals from all surveyed populations (Table 1). From each individual we collected one fresh flower from near to the top of the inflorescence and stored it in $70 \%$ ethanol for subsequent morphological analysis.

From a total of 126 individuals originating from close allopatric and sympatric populations, fresh leaves were also collected and stored at $-80^{\circ} \mathrm{C}(2005$ and 2006 samples) or in silica gel (2008 samples) for later molecular analyses.

In 2005, from the two sympatric populations, 20 individuals ( 12 from MR (Monti Rossi, Eastern Sicily), 8 from MA (Monte Arso, Eastern Sicily)) were randomly selected, excavated and later potted and cultivated under controlled conditions in an experimental area of the Botanic Gardens of Naples in order to perform crossing experiments. Hand-pollinations were performed after 1 year of controlled cultivation, after having observed that the trait variation of the two species and of their hybrids remained detectable also on cultivation.

\section{Molecular analyses}

Plant DNA was extracted from frozen or silica gel dried leaves using the cetyl trimethylammonium bromide method following Doyle and Doyle (1987) with minor modifications as reported in Moccia et al. (2007). Extracted DNAs were resuspended in $100 \mu \mathrm{l}$ of distilled water and DNA quality was examined both electrophoretically and with an Eppendorf BIO photometer (Eppendorf, Hamburg, Germany).

Genetic analyses were performed using amplified fragment length polymorphisms (AFLPs). AFLP procedure was performed with minor modifications, as described in Moccia et al. (2007) and using fluorescent dye-labelled primers. After a preliminary screening for variability and reproducibility of six primer pair combinations, three were chosen for this study (Eagc-Mcca, EagaMcgg and Eagg-Maac).

As the plastid genome is maternally inherited in orchids (Cafasso et al., 2005), plastid DNA variation was assayed in both species with the goal to 
Table 1 Sampled populations

\begin{tabular}{|c|c|c|c|c|c|c|}
\hline Population type & Taxon & Locality & Population ID & $\mathrm{n}$ (morphology) & n (molecular) & Region \\
\hline Close allopatric & A. morio & Novara di Sicilia & NS & 17 & 17 & Eastern Sicily \\
\hline Close allopatric & A. morio & Linguaglossa & LG & 17 & 16 & Eastern Sicily \\
\hline Distant allopatric & A. morio & Montebello & MB & 19 & - & Southern Italy \\
\hline Distant allopatric & A. morio & Matera & $\mathrm{TM}$ & 16 & - & Southern Italy \\
\hline Close allopatric & A. longicornu & Pennino di Lupo & $\mathrm{PL}$ & 16 & 16 & Eastern Sicily \\
\hline Close allopatric & A. longicornu & Siracusa & SR & 17 & 16 & Eastern Sicily \\
\hline Distant allopatric & A. longicornu & Korbous & KO & 40 & - & Northern Tunisia \\
\hline Distant allopatric & A. longicornu & El Haouaria & KA & 17 & - & Northern Tunisia \\
\hline Distant allopatric & A. longicornu & Monte Genuardo & MG & 22 & - & Western Sicily \\
\hline Distant allopatric & A. longicornu & Polizzi Generosa & $P G$ & 5 & - & Western Sicily \\
\hline Sympatric & A. morio/A. longicornu/hybrids & Monte Arso & MA & 47 & 39 & Eastern Sicily \\
\hline Sympatric & A. morio/A. longicornu/hybrids & Monti Rossi & MR & 44 & 22 & Eastern Sicily \\
\hline
\end{tabular}

characterize a diagnostic polymorphism that would allow to identify the maternal cytotype of hybrid individuals. First, a preliminary screening of plastid variation was carried out with a set of universal primers that amplify non-coding plastid DNA (Ebert and Peakall, 2009). PCR products were digested with a list of available restriction enzymes. From these tests, we selected a TaqI restriction site in the plastid rps2-rpoC2 spacer of A. longicornu that was not present in the corresponding region of A. morio, thus allowing to distinguish the two plastomes. All individuals from the close allopatric and sympatric populations were then analyzed in order to identify their maternal cytotype. PCR reactions were performed in a final volume of $25 \mu \mathrm{l}$ using $10 \mathrm{ng}$ template DNA, $200 \mu \mathrm{m}$ of each dNTP, 10 pmol of each of the two primers, $1 \mathrm{x}$ Taq buffer ( $50 \mathrm{~mm} \mathrm{KCl,} 10 \mathrm{~mm}$ Tris-HCl pH 9.0), $1.5 \mathrm{~mm} \mathrm{MgCl}_{2}$ and $0.5 \mathrm{U}$ of Taq polymerase (GE Healthcare, Chalfont St Giles, UK). The plastid locus was amplified with an initial denaturing step at $94^{\circ} \mathrm{C}$ for $3 \mathrm{~min}$, followed by 35 cycles of amplification at $94^{\circ} \mathrm{C}$ for $30 \mathrm{~s}, 57^{\circ} \mathrm{C}$ for $40 \mathrm{~s}$ and $72^{\circ} \mathrm{C}$ for $1 \mathrm{~min}$, with a final elongation step of $7 \mathrm{~min}$ at $72^{\circ} \mathrm{C}$. Five microliters of each PCR amplicon were digested with $5 \mathrm{U}$ of $\mathrm{TaqI}$ restriction enzyme at $50^{\circ} \mathrm{C}$ for $2 \mathrm{~h}$ and electrophoretically separated on a $2 \%$ agarose gel, stained with ethidium bromide and photographed on a UV transilluminator. A 100 base pair ladder (GE Healthcare) was used as molecular weight marker.

\section{Morphological analysis}

Flowers stored in $70 \%$ ethanol were dissected, and the main floral elements (labellum, spur and tepals) were mounted on a microscope slide and scanned. From the obtained images, floral traits were measured using IMAGEJ 1.33 (NIH, Bethesda, MD, USA). We investigated six morphological traits: left tepal length (YTepl), left tepal width (XTepl), spur length (YSpr), spur width (XSpr), labellum length $(Y L a b)$ and labellum width $(X L a b)$. More specifically, spur length was the distance between the spur mouth and the spur tip; spur width was the distance between the edges at mid spur length; labellum length was the distance between the labellum tip and the spur mouth; labellum width was the distance between the edges of the two lateral lobes (as reported in Moccia et al., 2007). These traits were chosen because they are either used to differentiate the two taxa or are potentially important for pollinator-mediated reproductive isolation between species.

\section{Natural pollination success and experimental crosses}

To estimate natural pollination success of plants in the field, we assessed flower number and fruit production from close allopatric populations of A. morio and A. longicornu, and from the sympatric populations. We defined individual pollination success as the proportion of fruits produced per inflorescence relative to the number of flowers.

To assess the presence and strength of postmating reproductive isolation, we performed experimental hand-pollinations between the 20 cultivated sympatric individuals during spring 2007, as described in Scopece et al. (2007). Following hand-pollinations, fruit maturation was monitored and ripe fruits were collected and stored in silica gel. Seed viability was assessed using an optical microscope and seeds were assigned to two categories, viable and inviable seeds, based on the presence or absence of embryos. As intraspecific controls, we used previously published experimental hand-pollinations reported in Moccia et al. (2007).

\section{Data analyses}

Different fragments in the AFLP fingerprinting patterns were treated as separate loci. AFLP fragments were scored with GENEMAPPER (Applied Biosystems, Foster City, CA, USA) and were treated as dominant marker loci with two states, presence (1) and absence (0), and coded to compose a binary data matrix.

The fixation index $\left(F_{\mathrm{ST}}\right)$ was used to estimate genetic differentiation among populations. We used the Bayesian approach proposed by Holsinger et al. (2002), as implemented in HICKORY v. 1.1 (http://darwin.eeb.uconn.edu/ hickory/software.html) using the $\mathrm{f}$ free model run to estimate $F_{\mathrm{ST}}$ for dominant AFLP markers.

To identify strongly differentiated loci between close allopatric A. morio and A. longicornu populations of the two species and to calculate $F_{\mathrm{ST}}$ values for each marker individually we used DFdist provided by MA Beaumont (available at http://www.rubic.rdg.ac.uk/ mab/stuff/), software that uses the method of Beaumont and Nichols (1996) with modifications for dominant markers.

Molecular hybrid indices were calculated for all individuals in the close allopatric and sympatric populations using HINDEX (Buerkle, 2005). The maximum likelihood hybrid index (HI) ranges between zero and one, corresponding to pure individuals of the alternative and reference species, respectively. In our analyses, plants with a HI ranging between 0 and 0.2 were assigned to $A$. longicornu, whereas individuals with $\mathrm{HI}$ between 0.8 and 1 were assigned to A. morio.

To estimate nuclear admixture proportions and patterns of introgression, we used the Bayesian clustering approach as implemented in STRUCTURE version 2.2 (Pritchard et al., 2000). Following the method described in Evanno et al. (2005), we selected $K=2$, corresponding to the assumption of two species contributing to the gene pool of the sample, after testing $K$ from 1 to 10 with a burn-in of 50000 steps followed by 300000 Markov chain Monte Carlo iterations with 10 replicas to confirm stabilization of the summary statistics. Estimations were carried out under the admixture model allowing for correlated allele frequencies on all sampled individuals without the consideration of sampling localities. We used DISTRUCT (Rosenberg, 2004) to graphically display the output obtained with STRUCTURE.

We also carried out the same analysis using only the two sympatric populations (that is, eliminating the close allopatric populations). As we found similar admixture patterns in the two sympatric populations (Figure 1) and no difference between the close allopatric populations of the same species, we merged them in the following analyses to enlarge the dataset.

In order to understand whether introgression for single markers deviates from neutral expectations, we estimated genetic clines (clines in genotype frequencies at individual loci as a function of $\mathrm{HI}$ ) for each locus in the admixed populations by using the close allopatric populations as reference samples. We performed this analysis using the parametric model, as implemented in 


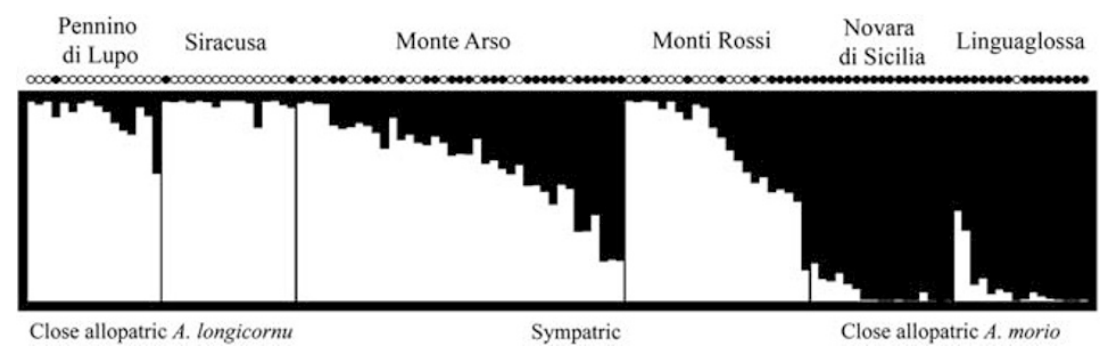

Figure 1 Proportional assignment of individual genotypes to each of the $K=2$ inferred clusters in the model-based admixture analysis. Black and white bars represent proportions of membership to the $A$. morio or $A$. longicornu clusters, respectively. Individuals are grouped by populations. Circles represent cytotypes of each individual (black circles $=$ A. morio; white circles=A. longicornu).

Table $2 F_{\mathrm{ST}}$ values ( \pm s.d.) among the investigated populations, calculated using Hickory v 1.1

\begin{tabular}{|c|c|c|c|c|c|c|}
\hline & $\begin{array}{l}\text { Close allopatric } \\
\text { A. morio (NS) }\end{array}$ & $\begin{array}{l}\text { Close allopatric } \\
\text { A. morio (LG) }\end{array}$ & $\begin{array}{l}\text { Close allopatric } \\
\text { A. longicornu }(S R)\end{array}$ & $\begin{array}{l}\text { Close allopatric } \\
\text { A. longicornu }(P L)\end{array}$ & Sympatric (MA) & Sympatric (MR) \\
\hline Close allopatric $A$. morio (NS) & - & $0.046( \pm 0.010)$ & $0.289( \pm 0.019)$ & $0.242( \pm 0.018)$ & $0.136( \pm 0.014)$ & $0.166( \pm 0.016)$ \\
\hline Close allopatric $A$. longicornu (SR) & - & - & - & $0.044( \pm 0.010)$ & $0.095( \pm 0.010)$ & $0.074( \pm 0.010)$ \\
\hline Close allopatric $A$. longicornu (PL) & - & - & - & - & $0.077( \pm 0.010)$ & $0.067( \pm 0.010)$ \\
\hline Sympatric (MA) & - & - & - & - & - & $0.032( \pm 0.007)$ \\
\hline
\end{tabular}

INTROGRESS (Gompert and Buerkle, 2009, 2010). Significance tests were based on 1000 permutations. In the absence of natural selection effects or of reproductive isolation mechanisms, all loci are expected to conform to neutral expectations (beside the effect of genetic drift or similar genetic mechanisms). A significant deviation would be interpreted as a signature of natural selection (either above or below the upper confidence interval) or of reproductive isolation (below the lower confidence interval) and, as evidence that a locus does not freely move across the admixed population.

Following Lexer et al. (2005), to test for the presence of cytonuclear disequilibrium across the sympatric zone, HI scores and plastid DNA haplotype data (a dichotomous variable with $0=A$. longicornu and $1=A$. morio) were compared using the nonparametric Spearman's rank correlation.

We used morphological traits to perform a canonical discriminant analysis describing the average floral morphology of each plant in the sympatric populations and to serve as an index of morphological variation (Aldridge, 2005). A stepwise method was applied with an F-value of 3.84 to enter a variable and $F$-value of 2.71 to remove it. Trait measurements from the parental populations were used to derive the discriminant function. Principal component analysis was calculated using SPSS (SPSS Inc., Chicago, IL, USA) and was used to explore patterns of morphological variation among the studied populations.

To detect significant differences between floral traits of the parental taxa and of the hybrids, we performed a Mann-Whitney $U$-test. For sympatric populations, assignment of individuals to three genotypic classes (A. morio, A. longicornu and hybrids) was achieved before the morphometric analysis using the AFLP-based HI scores. Only putative hybrids were used for comparison. We considered as putative hybrids all individuals with $\mathrm{HI}$ ranging between 0.2 and 0.8. This molecular-based taxon assignment was chosen to avoid circular reasoning during successive morphometric analysis.

HI scores were also used to assign individuals involved in experimental hand-pollinations to genotypes. Individuals were categorized as either parentals (HI either between 0 and 0.2 or between 0.8 and 1) or hybrids (HI between 0.2 and 0.8 ).

Pollination success (calculated as the proportion of fruits formed) was compared between close allopatric and sympatric populations using the nonparametric Mann-Whitney $U$-test. Phenotypic analysis was used to determine the relationships between traits and plant fitness. The correlation between an individual trait and plant pollination success is interpreted as evidence of selection (Lande and Arnold, 1983). In order to discern among directional and balancing selection, for those floral traits for which a significant correlation with pollination success was found, we tested whether a linear regression better fitted the data than a quadratic regression. For the same floral traits, we calculated coefficients of variance $(\mathrm{CV}=$ s.d./mean) in all sampled populations and tested whether differences were significant using Levene's test. For comparisons of percentages of viable and unviable seeds produced in different hand-pollinations, we used the nonparametric KruskalWallis Test.

All statistical analyses were performed using SPSS 13.0 (SPSS Inc.) and, for all tests, the significance threshold was set to 0.05 .

\section{RESULTS}

Molecular analyses

The three AFLP primer combinations produced a total of 118 fragments (26 with Eagc-Mcca, 42 with Eaga-Mcgg and 50 with Eagg-Maac; see Supplementary Table 1). The $F_{\mathrm{ST}}$ values indicate weak differentiation between close allopatric and sympatric populations, but higher genetic differentiation $\left(F_{\mathrm{ST}}>0.2\right)$ between parental A. morio and A. longicornu populations (Table 2).

$\mathrm{HI}$ calculated for close allopatric and sympatric populations were based on all available markers. Mean HI scores were 0.965 and 0.021 for close allopatric A. morio and A. longicornu, respectively, and mean HI scores for individuals from the two sympatric populations were 0.359 .

Bayesian admixture analyses indicated that the close allopatric populations of A. morio and A. longicornu that were used as reference populations were mainly composed of purebreds (mean proportion of membership in the two alternative clusters was 0.95 for close allopatric A. longicornu and 0.94 for close allopatric A. morio; Figure 1). The genotypes of both parental taxa contributed to the genotypes in the sympatric populations (Figure 1) but admixed individuals showed 

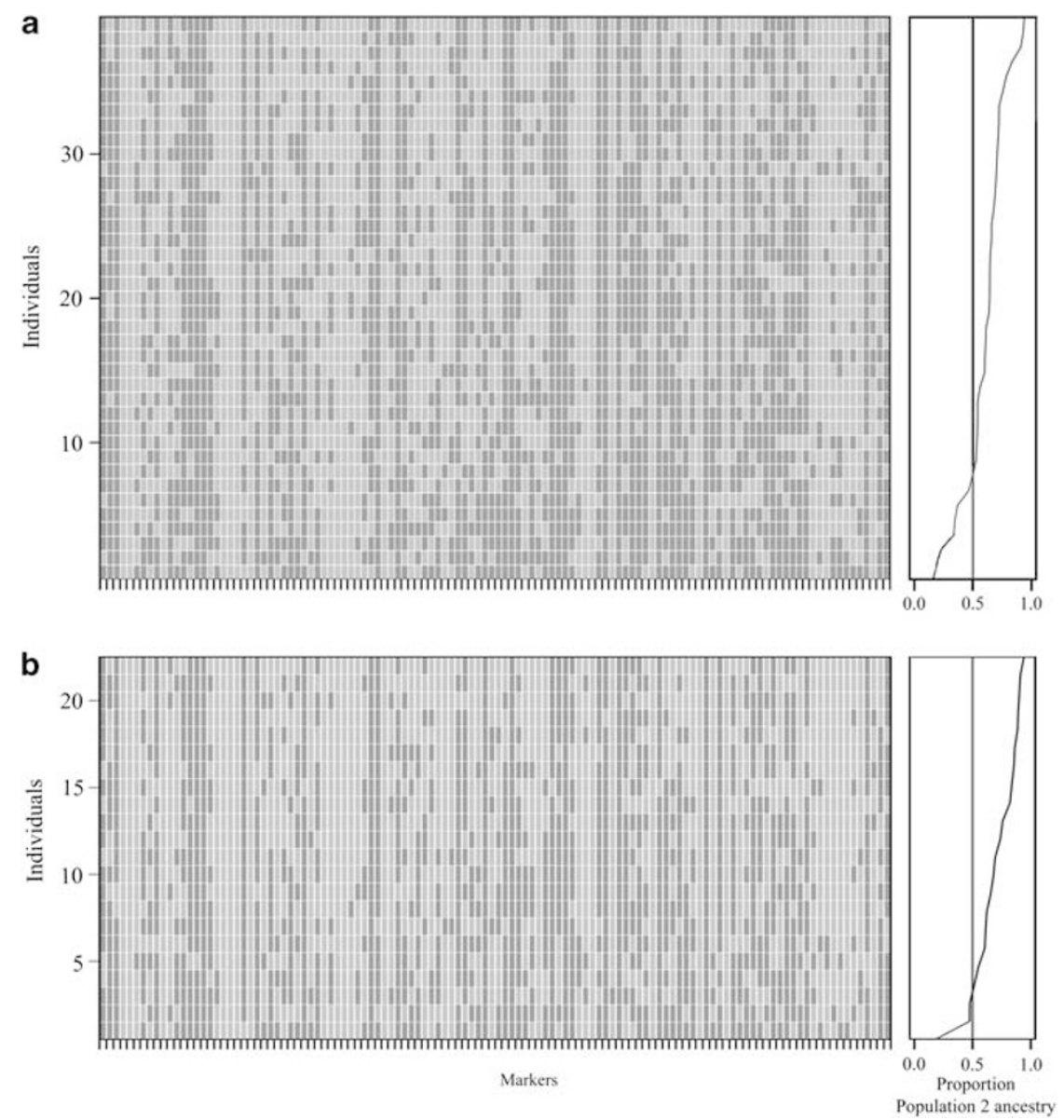

Figure 2 Overview plots of introgression for each marker and individual in the sympatric populations (a, population MA; b, population MR). Each rectangle indicates an individual's genotype at a given locus. $\mathrm{HI}$ of sympatric populations are given to the right. Individuals are sorted by increasing $\mathrm{HI}$ score.

a larger contribution of the A. longicornu genome (0.682 in MA population; 0.758 in MR population).

Estimates of genetic clines for each marker across the sympatric populations revealed that 18 out of the 118 available markers (15.3\%) significantly deviated from neutral expectations (Figures 2 and 4 and Supplementary Figure 1). Among the most informative loci $\left(F_{\mathrm{ST}}\right.$ higher than the third quartile), four markers significantly deviated from neutral expectations (Supplementary Figure 1).

Plastid DNA variation was assessed in individuals from close allopatric and sympatric populations. In the sympatric populations, 27 individuals carried the cytotype of $A$. longicornu (44.3\%) and the remaining 34 (55.7\%) individuals that of $A$. morio. By separating the individuals according to their HI scores, we found that most of the potential $\mathrm{F} 1$ individuals $(\mathrm{HI}=0.4-0.6)$ carried the $A$. morio cytotype (75\%). All potential backcrosses toward A. morio $(\mathrm{HI}=0.6-0.8)$ carried the A. morio cytotype, whereas backcrosses toward A. longicornu $(\mathrm{HI}=0.2-0.4)$ carried the cytotypes of $A$. morio $(51.7 \%)$ and of A. longicornu (48.3\%). From the sympatric populations, 3 out of the 13 potential $A$. longicornu individuals $(\mathrm{HI}=0-0.2)$ showed the cytotype of $A$. morio, while all the potential $A$. morio individuals $(\mathrm{HI}=$ $0.8-1)$ showed the expected cytotype. In the close allopatric populations of both taxa, most individuals carried the expected cytotype, with the exception of one individual of $A$. morio and three individuals of $A$. longicorn $u$ that possessed the alternative cytotype (Figure 1). The test for an association between nuclear genomic composition and plastid haplotype in individuals from the sympatric population was significant (Spearman's rho $=0.480, P<0.001$ ).

\section{Morphological analyses}

The principal component analysis based on the six morphological traits showed an overlap between the close allopatric and the sympatric populations and a weak differentiation between these populations and the distant allopatric populations (Supplementary Figure 3). Means and error bars of six morphological traits among putative hybrid genotypes (HI ranging between 0.2 and 0.8 ) from the sympatric populations and from the close and distant allopatric populations of A. morio and A. longicornu are reported in Figure 5. In the sympatric populations, we found that spur length (YSpr) was significantly negatively correlated with pollination success (Kendall's tau =$0.163 ; P=0.045)$. YSpr showed a significant linear regression with pollination success $\left(R^{2}=0.048 ; F=3.938 ; P=0.05\right)$, while quadratic regression was not significant $\left(R^{2}=0.048 ; F=1.951 ; P=0.149\right)$. In contrast, we did not find a significant correlation between this trait and pollination success in distant allopatric populations of both parental species. However, different populations exhibited different variability in spur length (distant allopatric $A$. morio: $\mathrm{CV}=0.13$; close allopatric $A$. morio: $\mathrm{CV}=0.11$; sympatric populations $\mathrm{CV}=0.17$; close allopatric A. longicornu: $\mathrm{CV}=0.18$; distant allopatric A. longicornu: $\mathrm{CV}=0.12$ ). Levene's test for the equality of variances showed that spur length variability was larger in sympatric populations than in close 


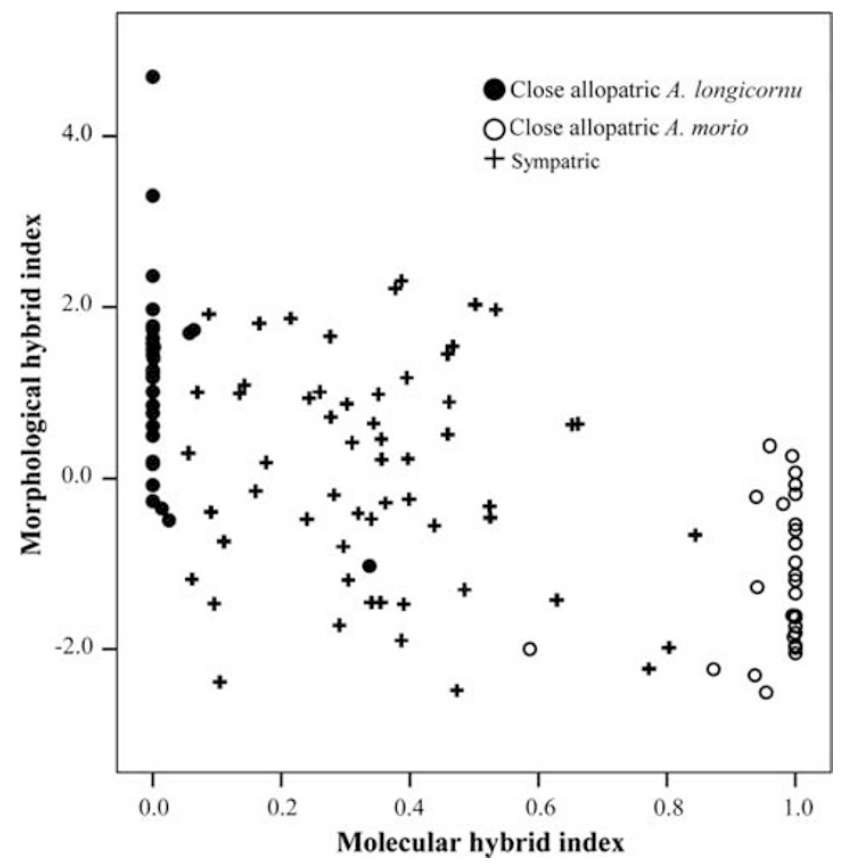

Figure 3 Correlation between molecular and morphological $\mathrm{HI}$ of close allopatric and sympatric populations of $A$. morio and A. Iongicornu.
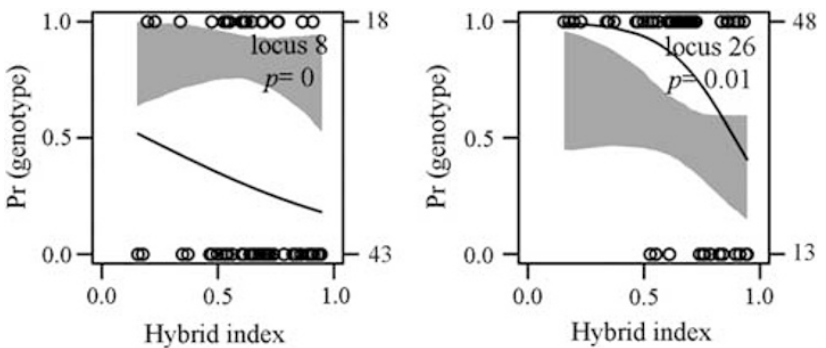

Figure 4 Genetic clines of two example loci (locus 8 and locus 26) that significantly deviate from neutral expectations. Locus names and $P$-values for the test of departure from neutrality are given in each plot. Solid lines represent the estimated clines while the grey regions indicate the 95\% confidence intervals for multinomial logistic regression fits to simulated data under the assumption of neutral introgression. Circles indicate the raw marker data (AFLP 'band absent' on top line and AFLP 'band present' along the bottom), with counts of each on the right vertical axis.

allopatric A. morio $(P=0.011)$ and in distant allopatric A. morio $(P=0.023)$. Similarly, spur length variability was larger, though not significantly so, in the sympatric populations than in the distant allopatric A. longicornu ( $P=0.686$ ) (Supplementary Figure 2). Only in close allopatric $A$. longicornu was spur length variability similar to that of the sympatric populations $(P=0.169)$.

The six morphological traits from individuals in the close allopatric and the sympatric populations were used as independent variables for the stepwise canonical discriminate analysis. The analysis produced the function (eigenvalue $=1.461, \chi^{2}=50.884, P<0.001$ ): $D=1.050$ $(Y S p r)-1.011(Y T e p l)+0.914(X T e p l)$. On the basis of this function, A. morio individuals received negative scores and A. longicornu positive scores in this sympatric population (A. morio $=-1.188$, A. longicornu=1.188). An index of morphological variation was calculated from the above formula for all individuals in the close allopatric and in the sympatric populations. We found a significant correlation between the index of morphological variation and the molecular HI (correlation coefficient $=0.771, P<0.001$; Figure 3).

\section{Natural pollination success and experimental crosses}

Mean natural pollination success in the sympatric populations $(15.3 \pm 1.8)$ was intermediate between close allopatric $A$. morio $(22.2 \pm 2.6)$ and A. longicornu $(8.6 \pm 2.3)$. Close allopatric A. morio populations achieved the highest pollination success (relative to sympatric: $U=1594.5, Z=-2.772, P=0.006$; relative to close allopatric A. longicornu: $U=549.0, Z=-4.840, P<0.001$ ); individuals in the sympatric populations had higher fruit production than those in the close allopatric A. longicornu populations $(U=2608.0, Z=-3.047$, $P=0.002$ ).

All 70 hand-pollinations performed between the 20 sympatric individuals produced fruits. All fruits contained a proportion of viable seeds (mean value $=71.6 \%$ ). By separating crossed individuals in parentals and hybrids, according to their HI scores, we found that the proportion of viable seeds produced did not differ among the potential combinations and from the intraspecific control $\left(\chi^{2}=4.909\right.$; $\mathrm{df}=3 ; P=0.179$; Table 3 ).

\section{DISCUSSION}

In this study, we investigated a secondary contact zone between two closely related Mediterranean food-deceptive orchids with similar karyotypes (D'Emerico et al., 1996) to assess whether floral trait differences that have evolved in allopatry are sufficient to confer reproductive isolation on secondary contact. Overall, we found that hybridization and strong genetic admixture occur in the two investigated sympatric populations, together with an apparent lack of postzygotic isolation mechanisms. These findings support the idea that these morphologically divergent but karyotypically similar taxa are collapsing into a hybrid swarm when in secondary contact and suggest that, in this case, allopatric divergence in floral traits alone is not sufficient to maintain species differences on sympatry. Instead, genomic divergence, for example, as a consequence of changes in ploidy level or the fixation of karyotype changes, are needed, as previously proposed (Cozzolino et al., 2004). Thus, in food-deceptive orchids, premating barriers are not sufficient for reproductive isolation in sympatry, as long as postmating barriers are weak or absent.

Our molecular and morphological investigation of two sympatric populations revealed strong genetic and phenotypic homogenization (Figure 1 and Supplementary Figure 3) in which parental categories are difficult to recognize. As shown in Figure 3, there is strong agreement between morphological and molecular data for the hybrids and for parental individuals from the close allopatric and sympatric populations. In contrast to other previously investigated hybrid zones between food-deceptive orchids, where a prevalence if not a dominance of F1 individuals was observed (Cozzolino and Widmer, 2005b; Moccia et al., 2007), we here found extensive introgression in the two sympatric populations to the extent that hybrid zones are dominated by backcross individuals (roughly $75 \%$ of hybrid individuals from the two sympatric populations; Figures 1 and 2).

Estimates of genetic differentiation $\left(F_{\mathrm{ST}}\right)$ were higher in comparisons between species (that is, between close allopatric A. morio and A. longicornu populations) than within species (that is, between two close allopatric populations of the same taxon). Overall, genetic differentiation $\left(F_{\mathrm{ST}}\right)$ among the studied populations was weak, indicating either ongoing gene flow or recent divergence of the two taxa. Interestingly, sequence divergence at neutral internal transcribed spacer loci between A. morio and A. longicornu is as high as between 

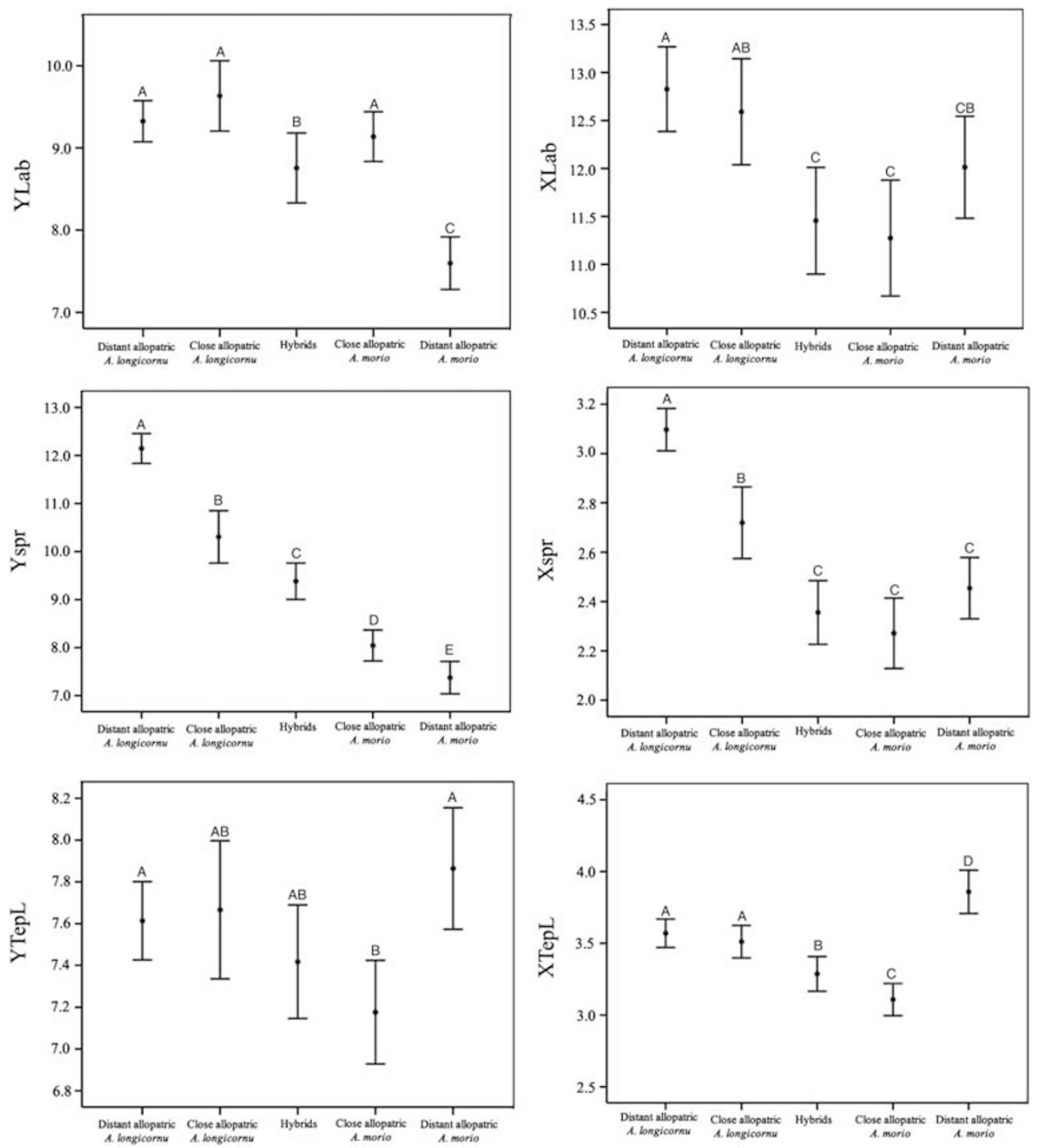

Figure 5 Means and standard errors for six floral traits in distant allopatric, close allopatric and hybrid individuals of $A$. morio and $A$. longicornu. Different letters indicate significant pairwise differences. YLab=labellum length; $\mathrm{XLab}=$ labellum width; YSpr=spur length; XSpr=spur width; YTepL=left tepal length; $\mathrm{XTepL}=$ left tepal width.

Table 3 Experimental crosses

\begin{tabular}{lccc}
\hline Cross type $^{\text {a }}$ & $N$ & Fruit production & Seed viability \\
\hline Interspecific & 10 & $100 \%$ & $61.3( \pm 7.3) \%$ \\
hybrid $\times$ hybrid & 43 & $100 \%$ & $76.0( \pm 2.3) \%$ \\
hybrid $\times$ parental & 17 & $100 \%$ & $71.9( \pm 5.3) \%$ \\
Intraspecific & 15 & $100 \%$ & $77.1( \pm 4.3) \%$
\end{tabular}

$\chi^{2}=4.909 ; \mathrm{df}=3 ; P=0.179$

andividuals are categorized according to their hybrid index $(\mathrm{HI})$ scores $(\mathrm{HI}=0-0.2$ and $\mathrm{HI}$ $0.8-1$ as putative parentals; $\mathrm{HI}=0.2-0.8$ as putative hybrids)

other reproductively well isolated food-deceptive species (Cafasso et al., 2001; Scopece et al., 2007). Although this comparison was based on a single gene and it is not uncommon for deep coalescence events to observe discordance among genetic regions in terms of levels of sequence divergence, high differentiation at internal transcribed spacer loci may suggests that the low $F_{\mathrm{ST}}$ values are unlikely due to recent divergence. Only few AFLP loci showed high $F_{\mathrm{ST}}$ values between the close allopatric populations of the two taxa and a single locus displayed a fixed difference. However, these loci displayed lower $F_{\mathrm{ST}}$ values between close allopatric and sympatric populations of the two taxa. Genomic cline estimates revealed that, in the admixed populations, 18 markers ( $15.3 \%$ of the total) significantly deviated from neutral expectations (Figures 2 and 4 and Supplementary Figure 1) suggesting that they may introgress less (or more) frequently than expected under neutrality (Rieseberg and Buerkle, 2002). Such a deviation can be interpreted as the signature of natural selection and suggests that marker movement across the hybrid zones is not fully random. Depending on the direction, deviation can represent either an increase in introgression rate (likely affecting loci that are ecologically advantageous in the hybrid zone; that is, Figure 4, locus 26) or a decrease in introgression rates (likely affecting loci that are ecologically disadvantageous or that interact epistatically; that is, Figure 4, locus 8). Among these deviating markers, we detected four (two below and two above neutral expectations) that are strongly differentiated 
between the close allopatric parental populations $\left(F_{\mathrm{ST}}\right.$ higher than the third quartile) and that could potentially represent the signature of divergent selection between the two taxa. These findings, however, have to be interpreted cautiously, because the investigated sympatric populations only encompassed few pure parental individuals and genetic clines were thus calculated using close allopatric populations as reference samples. Although these populations are growing in close proximity to the sympatric ones, they may differ in allele frequencies from those individuals that initiated the hybrid zones. Such differences could potentially bias estimates of confidence intervals of neutral introgression patterns. Particularly affected by such distortions can be loci for which the parental species data points are disconnected from the logistic regression lines (that is, the ones for which the regression line is not in contact with the data points at the extremes of the curve, see supplementary Figure 1).

In both investigated sympatric populations, introgression appears to be asymmetric, with $A$. longicornu contributing more to the genomic composition of hybrids than A. morio (Figure 1). Asymmetric introgression has often been reported in species pairs in which the relative abundance of parental species is unbalanced, because it is more likely that first-generation hybrids will mate with the more abundant parent (Lepais et al., 2009). In our study system, however, both parental species are about equally common. An alternative explanation for the observed asymmetric introgression may be the difference in spur length in the two parental species (longer in A. longicornu, shorter in A. morio, Figure 5), which may produce a preference in the pollinator set.

Characterization of the maternally inherited plastid genotypes in the sympatric populations revealed that both parental taxa contributed to the maternal cytotypes in the sympatric populations $(55.7 \%$ from A. morio, $44.3 \%$ from A. longicornu; Figure 1). However, plastid haplotypes were not equally distributed among hybrid classes. Most of the individuals with HI ranging from 0.4 to 0.6 (putative F1) carried the A. morio cytotype and all potential backcrosses toward A. morio carried the A. morio cytotype, indicating a cytonuclear disequilibrium. Ecological explanations (such as the difference in spur length or in phenology) can account for this strong asymmetry in cytotype representation in the hybrid individuals. Although not directly estimated, differences in flowering peaks (A. longicornu is slightly earlier flowering than $A$. morio) were observed. These cause pollen flow from A. longicornu to $A$. morio more often than vice versa and could account for the dominance of A. morio cytotypes in putative F1 hybrids. Consequently, backcrosses toward A. morio also carry the A. morio cytotype. In contrast, about half of the backcrosses toward A. longicornu carried the A. longicornu cytotype. These proportions suggest that putative F1 hybrids act both as male and female mating partners in backcrosses with A. longicornu. Alternatively, cytonuclear incompatibilities can shape the genetic structure of these hybrid zones. In fact, epistatic cytonuclear interactions could reduce the viability of plants carrying a genomic background of $A$. morio with an A. longicornu plastome producing the detected pattern of genetic introgression toward A. longicornu.

Our results from experimental hand-pollinations revealed full seed fertility in all possible crossing combinations involving parental species and hybrids (Table 3) thus indicating that, as in the majority of documented cases, if epistatic cytonuclear interactions exist, they are not expressed at the seed stage. Further studies are needed to understand whether the observed patterns of asymmetric introgression and cytonuclear disequilibrium are due to ecological factors or to epistatic cytonuclear interactions that affect later lifehistory stages.
Overall, comparisons between distant allopatric populations revealed an absence of marked phenotypic divergence between the two taxa (Supplementary Figure 3). However, pairwise comparisons of trait variation among populations revealed significant differences in some flower traits (Figure 5). In the contact zone, morphological traits of the hybrids (individuals with HI scores between 0.2 and 0.8 ) are mainly intermediate with respect to the parentals (Figure 5). Distant allopatric A. longicornu populations appeared morphologically similar to the close allopatric individuals (four out of six traits are not significantly different; Figure 5), while, on the contrary, we found large differences between the close allopatric and distant allopatric populations of A. morio (four out of six traits are significantly different; Figure 5). The large morphological trait differences between close and distant allopatric A. morio populations may be a consequence of the geographic distance separating these populations, which reduces gene flow and may lead to genetic drift. More likely, however, differences in local selective pressure (between the insular and the mainland populations) may account for the observed differences.

The two investigated taxa, A. morio and A. longicornu, differ in the length of their spurs (YSpr: Figure 5) and we found a significant correlation between spur length and pollination success in the sympatric populations, indicating directional selection on this trait. This finding may suggest that the primary element of divergence between these two taxa is differential adaptation to different pollinator sets (likely with different proboscis length) in allopatric populations. Unfortunately, no linkage mapping information is available to estimate ancestries and to account for linkage disequilibrium, and we were thus unable to directly associate this morphological trait with genetic markers. We can therefore at present not test whether trait divergence is associated with marker divergence or not.

The distribution of spur lengths in A. longicornu populations shows a displacement toward a smaller dimension in the Sicilian close allopatric populations when compared with the distant allopatric ones (supplementary Figure 2). This trend can be the effect of directional selection toward a shorter spur if the hymenopteran set in the sympatric population is mainly constituted by short-tongued bees but can also be a consequence of the high gene flow with the short-spurred A. morio. Furthermore, in distant allopatric populations, where we found little variation in spur length, we found no correlation between this trait and pollination success as opposed to the sympatric populations, where, compared with distant allopatric populations, the admixture of parental phenotypes increases trait variability (that is, coefficient of variance) on which pollinatormediated natural selection can act. The lack of a correlation between spur length and pollination success in allopatric populations can also be due to the effect of stabilizing selection, which in stable populations is difficult to detect (Inoue, 1986).

Ecological divergence is thought to be the primary cause of allopatric speciation, because it can directly (through the evolution of prezygotic barriers) or indirectly (through the fixation of BatesonDobzhansky-Muller incompatibilities) lead to the evolution of reproductive isolation (reviewed in Coyne and Orr, 2004). The evolution of premating incompatibility among allopatric plant populations is thought to be associated with the specialized relationships with pollinators (Stebbins, 1970). Pollinator-mediated divergent selection is thought to promote speciation in several specialized plant groups (Armbruster and Muchhala, 2009) but likely has limited relevance in generalized food-deceptive pollination systems. Plants that adopt this pollination strategy attract a wide range of occasional pollinators that, after a few trial visits, avoid the rewardless flowers and switch to rewarding ones (Gumbert, 2000). This insect behavior is expected to 
exert only weak selective pressure on floral traits (Salzmann et al., 2007; Juillet and Scopece, 2010) that is unlikely to produce effective premating barriers between species when in contact. In our study system, for instance, the difference in spur length (YSpr) and its correlation with pollination success may indicate that $A$. morio and $A$. longicornu have experienced divergent pollinator-mediated selection; however, our estimates of natural pollination success clearly showed that hybrid individuals from the sympatric populations are able to attract pollinators and achieve levels of pollination success that are not different from the mean of the parental taxa. This finding suggests that, as already reported for other food-deceptive species pairs (Cozzolino et al., 2006; Scopece et al., 2009), the combination of parental characteristics into the hybrid phenotypes does not prevent the attraction of pollinators and suggests that selection does not act against intermediate phenotypes. This confirms the weak role of pollinator-mediated selection in the evolutionary diversification of this orchid lineage.

Ecological divergent selection may indirectly promote the evolution of reproductive isolation through the accumulation of BatesonDobzhansky-Muller incompatibilities. The accumulation of these epistatic interactions can be accelerated if genes involved are under selection or are linked with genes under selection (Gavrilets, 2003). This process is time-dependent and in various animal and plant groups, orchids included, has been found to evolve in a clock-like fashion (for example, Scopece et al., 2007). Despite a degree of sequence divergence comparable to that of other reproductively isolated food-deceptive species (Cafasso et al., 2001; Scopece et al., 2007), we here found a nearly complete absence of fertility reductions at the postzygotic stage (Table 3 ). All experimental cross types led to the formation of a high proportion of viable seeds, without significant differences (Table 3) among them. In fact, the experimental design of the performed hand-pollinations, which involved pure parental individuals and hybrids (most likely of different generations), allowed us to exclude later stages of reproductive isolation such as partial hybrid sterility (likely at F1 and later generations) that has been reported to be among the first isolating mechanisms to evolve among closely related orchid species (Scopece et al., 2008). Moreover, all our handpollinations triggered the formation of fruits. This finding sharply contrasts with the common observation of postmating isolation mechanisms (such as pollen-stigma incompatibilities that impede fruit formation following interspecific pollinations, for example, Scopece et al., 2007) between sympatric food-deceptive orchid species. Such postmating isolation mechanisms have been proposed to evolve in secondary contact zones to avoid the waste of gametes because of interspecific matings (Dobzhansky, 1940), a common event among orchid species that share pollinators (Cozzolino and Widmer, 2005a).

\section{CONCLUSIONS}

In this study, we report that two Mediterranean food-deceptive orchid species with similar karyotypes collapse into a genetic and phenotypic continuum on secondary contact. Divergence in allopatry did thus not lead to the development of sufficiently strong reproductive isolation to prevent hybridization and introgression in sympatry.

Further studies are needed to understand why morphologically pure parental populations continue to exist in Sicily even in close proximity to hybrid zones. A potential explanation could be that insufficient time has elapsed since the two lineages have come into secondary contact. Sicily is part of the original distribution area of A. longicornu, while it represents the southern distribution limit of A. morio. Eastern Sicily can thus be a recent meeting point between these two taxa as a consequence of climatic oscillations linked to recent glacial periods
(Zitari et al., 2011). Alternatively, natural selection against particular parental genome combinations in hybrids brought about by negative epistatic interactions among gene complexes that have diverged in allopatry can limit the spread of the hybrid swarm and prevent complete genetic admixture of the two parental species.

The apparent absence of reproductive isolation between A. morio and A. longicornu sharply contrasts with the multiple postmating (preand postzygotic) barriers that have been reported in other fooddeceptive orchid species pairs with similar degrees of sequence divergence. However, these other food-deceptive species pairs frequently show strong karyotype asymmetries, which suggests that in Mediterranean food-deceptive species, allopatric ecological divergence of floral traits alone has only a limited role in the speciation process.

\section{DATA ARCHIVING}

Data have been deposited at Dryad: doi:10.5061/dryad.7pd8q.

\section{CONFLICT OF INTEREST}

The authors declare no conflict of interest.

\section{ACKNOWLEDGEMENTS}

We thank A Cristaudo for kindly sharing information on orchid distribution in Sicily. We also thank V Tranchida-Lombardo, F Pinheiro, C Palma-Silva and D Cafasso for help with molecular analyses and three anonymous referees for providing comments that significantly improved the paper. This study was funded by the Programma di Rilevante Interesse Nazionale (PRIN 2007).

Aldridge G (2005). Variation in frequency of hybrids and spatial structure among Ipomopsis (Polemonaceae) contact sites. New Phytol 167: 279-288.

Armbruster WS, Muchhala N (2009). Associations between oral specialization and species diversity: cause, effect, or correlation? Evol Ecol 23: 159-179.

Barton NH, Hewitt GM (1985). Analysis of hybrid zones. Annu Rev Ecol Syst 16: 113-148.

Beaumont MA, Nichols RA (1996). Evaluating loci for use in the genetic analysis of population structure. Proc $R$ Soc Lond B Biol Sci 263: 1619-1626.

Bomblies K, Weigel D (2007). Hybrid necrosis: autoimmunity as a potential gene-flow barrier in plant species. Nature Rev Genet 8: 382-393.

Buerkle CA (2005). Maximum-likelihood estimation of a hybrid index based on molecular markers. Mol Ecol Notes 5: 684-687.

Cafasso D, Pellegrino G, Caputo P, Scrugli A, Cozzolino S (2001). Genetic relatedness of insular segregates of Mediterranean orchid species as inferred from ITS sequences analysis. Bot Helv 111: 181-190.

Cafasso D, Widmer A, Cozzolino S (2005). Chloroplast DNA inheritance in the orchid Anacamptis palustris using single-seed polymerase chain reaction. J Hered 96: 66-70. Coyne JA, Orr HA (2004). Speciation. Sinauer Associates: Sunderland, MA.

Cozzolino S, D'Emerico S, Widmer A (2004). Evidence for reproductive isolate selection in Mediterranean orchids: karyotype differences compensate for the lack of pollinator specificity. Proc $R$ Soc Lond B Biol Sci 271: 259-262.

Cozzolino S, Nardella AM, Impagliazzo S, Widmer A, Lexer C (2006). Hybridization and conservation of Mediterranean orchids: should we protect the orchid hybrids or the orchid hybrid zones? Biol Cons 129: 14-23.

Cozzolino S, Scopece G (2008). Specificity in pollination and consequences for reproductive isolation in deceptive Mediterranean orchids. Philos Trans R Soc Lond B Biol Sci 363: 3037-3046.

Cozzolino S, Widmer A (2005a). Orchid diversity: an evolutionary consequence of deception? Trends Ecol Evol 20: 487-48. 494.

Cozzolino S, Widmer A (2005b). The evolutionary basis of reproductive isolation in Mediterranean orchids. Taxon 54: 977-985.

Delforge P (2005). Orchids of Europe, North Africa and the Middle East. A\&C Black: London, UK.

D'Emerico S, Pignone D, Bianco P (1996). Karyomorphological analyses and heterochromatin characteristics disclose phyletic relationships among $2 n=32$ and $2 n=36$ species of Orchis (Orchidaceae). Plant Syst Evol 200: 111-124.

Dobzhansky T (1940). Speciation as a stage in evolutionary divergence. Am Nat 74: 312-321.

Doyle JJ, Doyle JL (1987). A rapid DNA isolation procedure from small quantities of fresh leaf tissues. Phytochem Bull 19: 11-15.

Ebert D, Peakall R (2009). A new set of universal de novo sequencing primers for extensive coverage of noncoding chloroplast DNA: new opportunities for phylogenetic studies and cpSSR discovery. Mol Ecol Resources 9: 777-783. 
Evanno G, Regnaut S, Goudet J (2005). Detecting the number of clusters of individuals using the software STRUCTURE: a simulation study. Mol Ecol 14: 2611-2620.

Gavrilets S (2003). Models of speciation: what we have learned in 40 years? Evolution 57: 2197-2215.

Gompert Z, Buerkle CA (2009). A powerful regression-based method for admixture mapping of isolation across the genome of hybrids. Mol Ecol 18: 1207-1224.

Gompert Z, Buerkle CA (2010). INTROGRESS: a software package for mapping components of isolation in hybrids. Mol Ecol Resources 10: 378-384.

Grant V (1981). Plant speciation. Columbia University Press: New York, NY.

Gumbert A (2000). Color choices by bumble bees (Bombus terrestris): innate preferences and generalization after learning. Behav Ecol Sociobiol 48: 36-43.

Harrison RG (1993). Hybrid Zones and the Evolutionary Process. Oxford University Press: New York.

Hewitt GM (2001). Speciation, hybrid zones and phylogeography - or seeing genes in space and time. Mol Ecol 10: 537-549.

Hewitt GM (2004). Genetic consequences of climatic oscillations in the Quaternary. Philos Trans R Soc Lond B Biol Sci 359: 183-195.

Holsinger KE, Lewis PO, Dey DK (2002). A Bayesian approach to inferring population structure from dominant markers. Mol Ecol 11: 1157-1164.

Inoue K (1986). Experimental studies on male and female reproductive success: effects of variation in spur length and pollinator activity on Platanthera mandarinorum ssp. hachijoensis (Orchidaceae). Plant Sp Biol 1: 207-215.

Juillet N, Scopece G (2010). Does floral trait variability enhance reproductive success in deceptive orchids? Perspect Plant Ecol Evol Syst 12: 317-322.

Lande R, Arnold SJ (1983). The measurement of selection on correlated characters. Evolution 37: 1210-1226.

Lepais O, Petit RJ, Guichoux E, Lavabre JE, Alberto F, Kremer A et al. (2009). Species relative abundance and direction of introgression in oaks. Mol Ecol 18: 2228-2242.

Lexer C, Fay MF, Joseph JA, Nica MS, Heinze B (2005). Barrier to gene flow between two ecologically divergent Populus species, P.alba (white poplar) and P. tremula (European aspen): the role of ecology and life history in gene introgression. $\mathrm{Mol} \mathrm{Ecol}$ 14: 1045-1057.
Moccia MD, Widmer A, Cozzolino S (2007). The strength of reproductive isolation in two hybridizing food-deceptive orchid species. Mol Ecol 16: 2855-2866.

Pritchard JK, Stephens M, Donnelly P (2000). Inference of population structure using multilocus genotype data. Genetics 155: 945-959.

Rieseberg LH, Buerkle C (2002). Genetic mapping in hybrid zones. Am Nat 159: 37-49. Rieseberg LH, Willis JH (2007). Plant speciation. Science 317: 910-914.

Rosenberg NA (2004). DISTRUCT: a program for the graphical display of population structure. Mol Ecol Notes 4: 137-138.

Rundle HD, Nosil P (2005). Ecological speciation. Ecol Lett 8: 336-352.

Salzmann CC, Nardella AM, Cozzolino S, Schiestl FP (2007). Variability in floral scent in rewarding and deceptive orchids: the signature of pollinator-imposed selection? Ann Bot 100: 757-765.

Schluter D (2000). The Ecology of Adaptive Radiation. Oxford University Press: Oxford, UK.

Scopece G, Juillet N, Müller A, Schiestl FP, Cozzolino S (2009). Pollinator attraction in Anacamptis papilionacea (Orchidaceae): a food or a sex promise? Plant Sp Biol 24: 109-114.

Scopece G, Lexer C, Widmer A, Cozzolino S (2010). Polymorphism of postmating reproductive isolation within plant species. Taxon 59: 1367-1374.

Scopece G, Musacchio A, Widmer A, Cozzolino S (2007). Patterns of reproductive isolation in Mediterranean deceptive orchids. Evolution 61: 2623-2642.

Scopece G, Widmer A, Cozzolino S (2008). Evolution of postzygotic reproductive isolation in a deceptive orchid lineage. Am Nat 171: 315-326.

Stebbins GL (1970). Adaptive radiation of reproductive characteristics in angiosperms. I. Pollination mechanisms. Ann Rev Ecol Syst 1: 307-326.

Taylor EB, Boughman JW, Groenenboom M, Sniatynski M, Schluter D, Gow JL (2006). Speciation in reverse: morphological and genetic evidence of the collapse of a three-spined stickleback (Gasterosteus aculeatus) species pair. Mol Ecol 15 : 343-355.

Zitari A, Tranchida Lombardo V, Cafasso D, Helal HN, Scopece G, Cozzolino S (2011). The disjoint distribution of Anacamptis longicornu in the West-Mediterranean: the role of vicariance versus long-distance seed dispersal. Taxon (in press).

Supplementary Information accompanies the paper on Heredity website (http://www.nature.com/hdy) 